\title{
Performance Analysis of Clustering Based Image Segmentation Techniques
}

\author{
Dr. Samuel Manoharan, \\ Professor, \\ Department of Electronics, \\ Bharathiyar College of Engineering and Technology, \\ India. \\ Email: jsamuel@bcetedu.in
}

\begin{abstract}
As the images are examined using the latest machine learning process, the techniques for computing the images become highly essential. This computation applied over the images allows one to have an assessable information's or values from the images. Since segmentation plays a vital role in processing of images by enhancing or hypothetically altering the images making the examination of valuable insights easier. Several procedures and the methods for segmenting the images have been developed. However to have an better process it is important to sort out an effective segmentation procedure, so the paper performs the analysis of the clustering based image segmentation techniques applied on the magnetic resonance image of the human brain to detect the white matter hyper intensities part. The evaluation process take place in the MATLAB to evince the accurate valuation procedure. The optimal procedure is sorted out to be used in observing and examining the medical images by implementing over a computer assisted tool.
\end{abstract}

Keywords: Image Processing, Segmentation, Human Brain, White Matter Hyper Intensities Part, Accuracy, Clustering

\section{Introduction}

The digital image processing enables one to have a required insights and the data when an image is tested. In other words the image processing enables one to have the interpretation of the images that are computed by it. Fundamentally these image processing techniques function on a two dimensional square that is known as the pixel. The extraction of valuable information from an image using the image processing techniques involves several stages of processing. The segmentation is also has a significant part in the computation of image and in the computer vision as it makes the analyzing process easier by enhancing and potentially varying the images to digital form.

Basically segmentation is not just a simple procedure, it includes and executes multiple of chores. The images that are lay open to the process of segmentation are segregated into manifold divisions, and the 
Journal of Innovative Image Processing (JIIP) (2020)

Vol.02/ No. 01

Pages: $14-24$

https://www.irojournals.com/iroiip/

DOI: https://doi.org/10.36548/jiip.2020.1.002

valuable insights would be obtained as a result either as texture, intensity or color. In addition from this the segmentation take an important part in recognizing the images.

Several techniques are available and more new techniques are devised to perform the procedures of segmentation. But the available and the devised methodologies prove to be less efficient as they are practically incompatible over all sorts of images. So there arises a necessity to identify an effective segmentation procedure, to enhance the performance of the image processing.

The segmentation procedure scopes in simplifying the examination process into eloquent and easily analyzable form. It is vital and the initial procedure in the analyzing an image. The main purpose of the segmentation process is to segregate the images to have the similar attributes. It is essential first step in many of the application like object detection and recognition, retrieval of image based on the content, video surveillance, medical imaging etc. Apart from this the segmentation is the first and the foremost step in developing a multi label classifier construction to classify more than one object found in the image. For this it works by segregating the image into multiple sections and groups the same attributes pixels and creates a pixel-wise mask for all the objects in the image. The figure. 1 below shows the stages in the segmentation process.

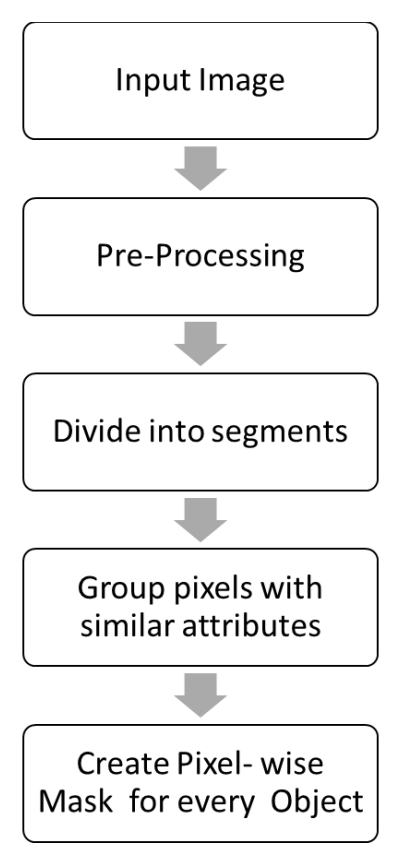

Figure.1Segementation Procedure 
Journal of Innovative Image Processing (JIIP) (2020)

Vol.02/ No. 01

Pages: $14-24$

https://www.irojournals.com/iroiip/

DOI: https://doi.org/10.36548/jiip.2020.1.002

Basically the segmentation process could be classified into two fundamental kinds as local and global segmentation and further based on the image properties as discontinuity detection strategy and similarity detection strategy. The former types of segmentations mentioned are concerned about the particular section or the area of an image and whole image that is encompassed with the huge set of pixels. The later segmentation types based on the image properties segments the images into regions based on the discontinuity and similarity respectively. The several segmentation methods of the image are listed below in figure. 2

There are more than a few prevailing practices that are utilized for segmenting images. These methods have their own significance. These modus operandi could come within reach of from dualistic elementary methods of segmentation i.e. region based or the edge based, every single procedures can be applied on dissimilar images to accomplish the necessary segmentation. All these techniques could be classified based on three categories. As (i) structural: works on the structure of the region, (ii) Stochastic: works on the discrete pixel values and (iii) hybrid: Combines both the structural and the stochastic.

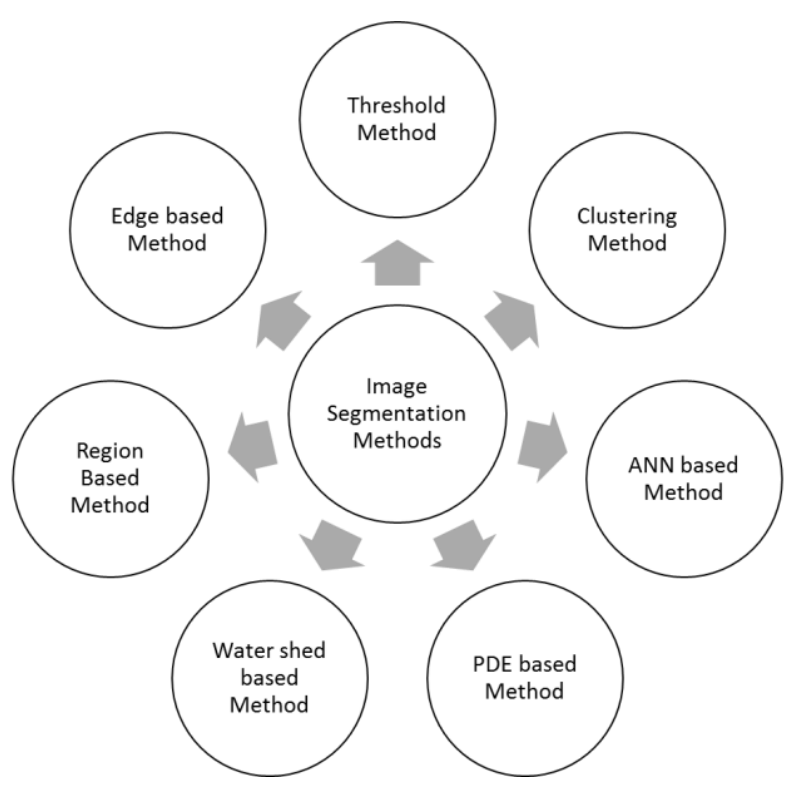

Figure.2 Image Segmentation Methods

As the digital images acquired are projected to multitudes of distortions that deteriorates the quality of the image, an effective segmentation process becomes a necessary requisite for retaining the valuable data of the images especially in medical imaging. 
The paper devise the performance analysis of the hard and soft clustering methods to sort out the efficient one to be used in observing and examining the medical images by implementing over a computer assisted tool. The proposed method aims in applying the clustering method of segmenting over the images of the human brain to identify the white matter hyper intensities form the magnetic resonance images to have an early diagnosis of the serious illness caused by the small vessel diseases and elude it.

The performance analysis process carried out is subdivided into many section as literature survey in segment two, methodology used in segment three the result analysis in segment four and conclusion in segment 5 .

\section{Literature Survey}

Heena, A., $\mathrm{N}$ et al [1] organized a new strategy to view the variety of techniques used in segmenting the images. Papadopoulos, et al [2] proposed a community detection based on the graph based clustering to elude the overload issue endured while sharring photos Brickman et al [3] presented the methods of testing and preserving the cognition and the white matter hyper intensities Suganya, R., et al [4] present the " review on fuzzy c means"

Ajala Funmilola, A. et al [5] devised the method of segmenting incorporating the fuzzy c and K means clustering. Wardlaw, et al [6] elaborated the complete particulars of the components present in W-M-H-I Jeyavathana, $\mathrm{R}$ et al [7] has presented the "analysis on preprocessing and segmentation techniques for medical images" Isa, et al [8] conducted a . "A new technique for K-means cluster centers initialization of WMH segmentation."

Koresh, M. H et al [9] presented the "Computer vision based traffic sign sensing for smart transport." Chandy, Abraham et al [10] elaborated the "A Review on Iot Based Medical Imaging Technology for Healthcare Applications." Bindhu, et al [11] presents the "Biomedical Image Analysis Using Semantic Segmentation." V Kumar, T. Senthil et al [12] proposed the "A Novel Method for HDR Video Encoding, Compression and Quality Evaluation."

\section{Methodology}

In general the W-M-H-I (the white matter hyper intensities) present in the human brain could be identified using the magnetic resonance images scanning. They lay deep inside the white matter of the brain and 
would cause serious illness and damages in the brain if not detected in its early stage. This causes the small vessel disease affecting the people in the adolescent and the old ages leading to serious sickness such as the Alzheimer, Dementia, Stroke and Cognitive. So this makes it requisite for an effective method to process the medical images in order to have a perfect diagnosis. The proposed method utilizes hybridized hard and soft clustering based segmentation to acquire a valuable information and data related to the white matter, and the performance is analyzed with the existing methods based on the accuracy measure of the identifying the region of W-M

\subsection{Data Set Used}

The procedure of segmenting is carried out in the "T2 weighted Magnetic Resonance Images" (T2-WI) that are acquired from the advanced medical research institutes of the world. From enormous of images data set gathered fifteen to twenty slices of images were extracted for each type of brain based diseases and totally hundred images were tested under the proposed method of segmenting.

\subsection{Segmentation}

The segmentation process as stated in figure. 1 starts with the preprocessing stage to separate the noises present in its back ground. In general the removing the noises and enhancing the quality of the images are done in the preprocessing stages. The proposed method employs the filtering and the morphological operations for removing the noises in the images. The images acquired in the gray scale is transformed into images of binary form to perform the technique of segmentation, each pixel in the image in the form of gray scale is substituted with the eight bit gray scale level as 000 and 111 for the white and black areas respectively. Hence the properties of the image minimizes the difficulties in the analyzing stage as intensity values of every pixel in the images are provided.

To further improvise the examination process, a structural breakdown is followed by dividing the images according to the ROI (region of interest). The tool boxes are encompassed with manifold shapes to be utilized as the region of interest, but the proposed method utilizes the MATLAB image processing tools to perform the morphological operations. These strides removed the scalp and the skull of the magnetic resonance imaging of the human brain acquired. By this the boundaries were eroded on the pixels of the images. The preprocessing method is examined by applying it all through the segmentation process.

\subsection{Hard and Soft Based Segmentation Process}


The segmentation process has become very important in the image processing as it lays as the very significant process in many application such as image retrieval, classification and the object detection etc. the completion of the preprocessing leads to the segmentation of the images, the proposed method utilizes the hard (K-means) and soft (Fuzzy C means) clustering to identify the regions with similarity and group them and Super pixels algorithm to cluster the images and view the segmented images clearly. The images clustered are converted in RGB format to have the regions of interest and every regions of interest that is clustered is estimated by combining the properties such as the color and the value of the intensities.

\subsubsection{Hard Clustering Based Segmentation}

This simple clustering segmentation segregates the images into set of clusters so that one pixel is member of only one the cluster. The membership function represented either as zero or one. Where one represents the presence of the pixel in the cluster and the zero represents its absence. The K-means clustering is a hard clustering method, it is an unsupervised learning algorithm with the very complexities in the computation. The cluster numbers are decided by the users.

\section{Algorithm}

a. The centers are computed based on the closest intensities.

b. The centroid values are set randomly.

c. Pixels are assigned to the nearest center

d. Given 'n' number of clusters centers, ' $K$ ' number of pixels.

e. The Euclidean distance $\left(\|x i-y j\|^{\wedge} 2\right.$ ) between the pixel and the nearest center is evaluated.

f. The process is continued for number of iterations to reduce the centroid distance in the middle of the points.

g. Each iteration is allotted new point of location to the nearest cluster centroid.

$\mathrm{h}$. The process is repeated until there are no changes in the values.

The equation. 1 below gives the computation process of the k-means algorithm.

$$
\operatorname{Dist}_{y}=\sum_{j=1}^{k} a \sum_{i=1}^{n}\left(\|x i-y j\|^{\wedge} 2\right)
$$

\subsubsection{Soft Clustering Based Segmentation}


It is a natural type of clustering, in which the segregation is done using the partial membership i.e. "one pixel belong to more than one clusters and the degree of the each pixel is defined using the member ship values. It is more flexible technique than the other techniques. Fuzzy c means is the popular example for the soft clustering based segmentation.

\section{Algorithm}
a. Select cluster center 'C'.
b. Estimate the fuzzy member ship using equation. 2
c. Update the fuzzy centers ' $F_{c}{ }^{\prime}$ using equation .3 for $F_{c}^{\prime}=1,2, \ldots . C$
d. The process is continued for ' $\mathrm{K}$ ' iterations and $M_{f}$ is the member ship function.
e. The procedure allots member ship to all the data based on the clusters.

The Equation. 2 and 3 gives the mathematical equation for computing the member ship function and updating the ' $F_{c}$ '

$$
\begin{aligned}
& M_{f}=\frac{1}{\sum_{k=1}^{c}\left(\frac{\text { dist } i j}{\text { dist } i k}\right)\left(\frac{2}{m^{-1}}\right)} \\
& F_{C}=\frac{\sum_{i=1}^{n} M_{f} m_{* x i}}{\sum_{i=1}^{n} M_{f} m}
\end{aligned}
$$

The proposed method combines both the hard and soft techniques of segmentation to have a perfect grouping of similar pixels, to preserve more information of the actual image. The perfect grouping of similar pixels is achieved using the $\mathrm{k}$-means that performs repeated re-estimation until the no changes are incurred and the preserving information's of the actual images are done using the fuzzy C means. Further the super pixel employed creates a pixel wise masks for each group of pixels to retain the quantitative details of the actual image and obtain best result.

\section{Results Analysis}

The results obtained on testing the proposed method with the slices of images obtained from the advanced medical institute, all over the world, is presented in the section. The results providing both the qualitative 
Journal of Innovative Image Processing (JIIP) (2020)

Vol.02/ No. 01

Pages: $14-24$

https://www.irojournals.com/iroiip/

DOI: https://doi.org/10.36548/jiip.2020.1.002

and the quantitative measure are acquired and further the accuracy of existing segmentation techniques and the proposed method are compared.

The evaluation process completely takes place using the MATLAB image processing tools. The qualitative estimation is done based on the envisage, the process of segmenting is done to identify the W-M-H-I in the brain of humans. So regions of the brain are clustered into four groups as white matter, background, grey matte and the cerebrospinal fluid. To have a better analysis the images are transformed into gray scale as every pixel in the gray scale representation is depicted only using a single value. The results below in figure. 3 provide the output achieved through the proposed segmentation combining the k-means, Fuzzy C means and the super pixel

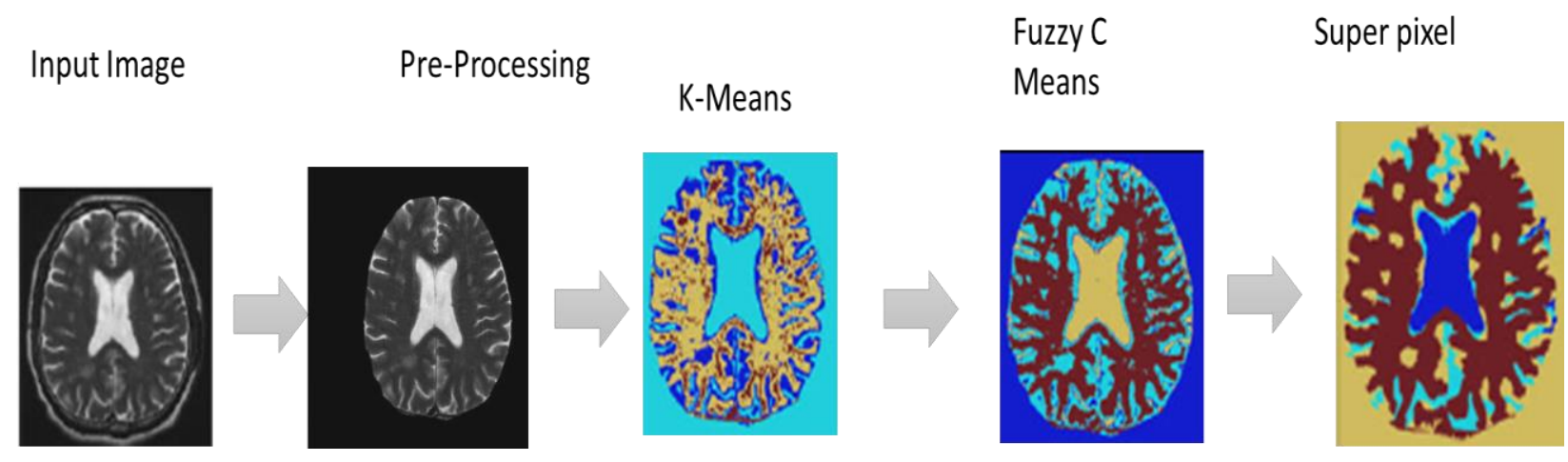

Figure.3 Qualitative Results of Proposed Clustering

The following table. 1 presents the quantitative results of the segmentation process, presenting the values of the segmented area, the mean intensity and the average time required in processing. The k-means integrated with the fuzzy c means and the super pixel $(\mathrm{KmFcSp})$ is compared with the process of $\mathrm{K}$-means, Fuzzy $\mathrm{C}$ means and the super pixel. 
Journal of Innovative Image Processing (JIIP) (2020)

Vol.02/ No. 01

Pages: $14-24$

https://www.irojournals.com/iroiip/

DOI: https://doi.org/10.36548/jiip.2020.1.002

\begin{tabular}{|l|l|c|c|l|}
\hline Region & Techniques & Segmented Area & Mean Intensity & $\begin{array}{l}\text { Average time } \\
\text { of Processing } \\
(\mathrm{ms})\end{array}$ \\
\hline \multirow{4}{*}{ W-M-H-I } & K-Means & 10.67 & 83.24 & 36.456 \\
\cline { 2 - 5 } & Fuzzy- C-Means & 145.9 & 65.675 & 37.89 \\
\cline { 2 - 5 } & KmFcSp & 250.45 & 77.589 & 19.455 \\
\hline \multirow{3}{*}{ WM } & K-Means & 11000 & 59.456 & 23.45 \\
\cline { 2 - 5 } & Fuzzy- C-Means & 3000 & 87.3345 & 21.56 \\
\cline { 2 - 5 } & KmFcSp & 34000 & 53.23 & 14.567 \\
\hline \multirow{3}{*}{ GM } & K-Means & 1800 & 1770.678 & 18.245 \\
\cline { 2 - 5 } & Fuzzy- C-Means & 1850 & 135.78 & 17.567 \\
\cline { 2 - 5 } & KmFcSp & 24,242 & 92,45 & 12.578 \\
\hline \multirow{3}{*}{ CSF } & K-Means & 400 & 328.432 & 18.865 \\
\cline { 2 - 5 } & Fuzzy- C-Means & 800 & 129.456 & 19.548 \\
\cline { 2 - 5 } & KmFcSp & 2500 & 87.34 & 10.45 \\
\hline
\end{tabular}

Table.1 Quantitative Results

The figure. 4 below depicts the accuracy measure of the proposed algorithm and the existing algorithms in identification of the similarities in the regions of human brain to locate the white- matter- hyper-intensities the proposed method is compared with the k-means and the fuzzy $\mathrm{C}$ means. The accuracy measure of the proposed method seems to outperform the other two methods. 
Journal of Innovative Image Processing (JIIP) (2020)

Vol.02/ No. 01

Pages: $14-24$

https://www.irojournals.com/iroiip/

DOI: https://doi.org/10.36548/jiip.2020.1.002

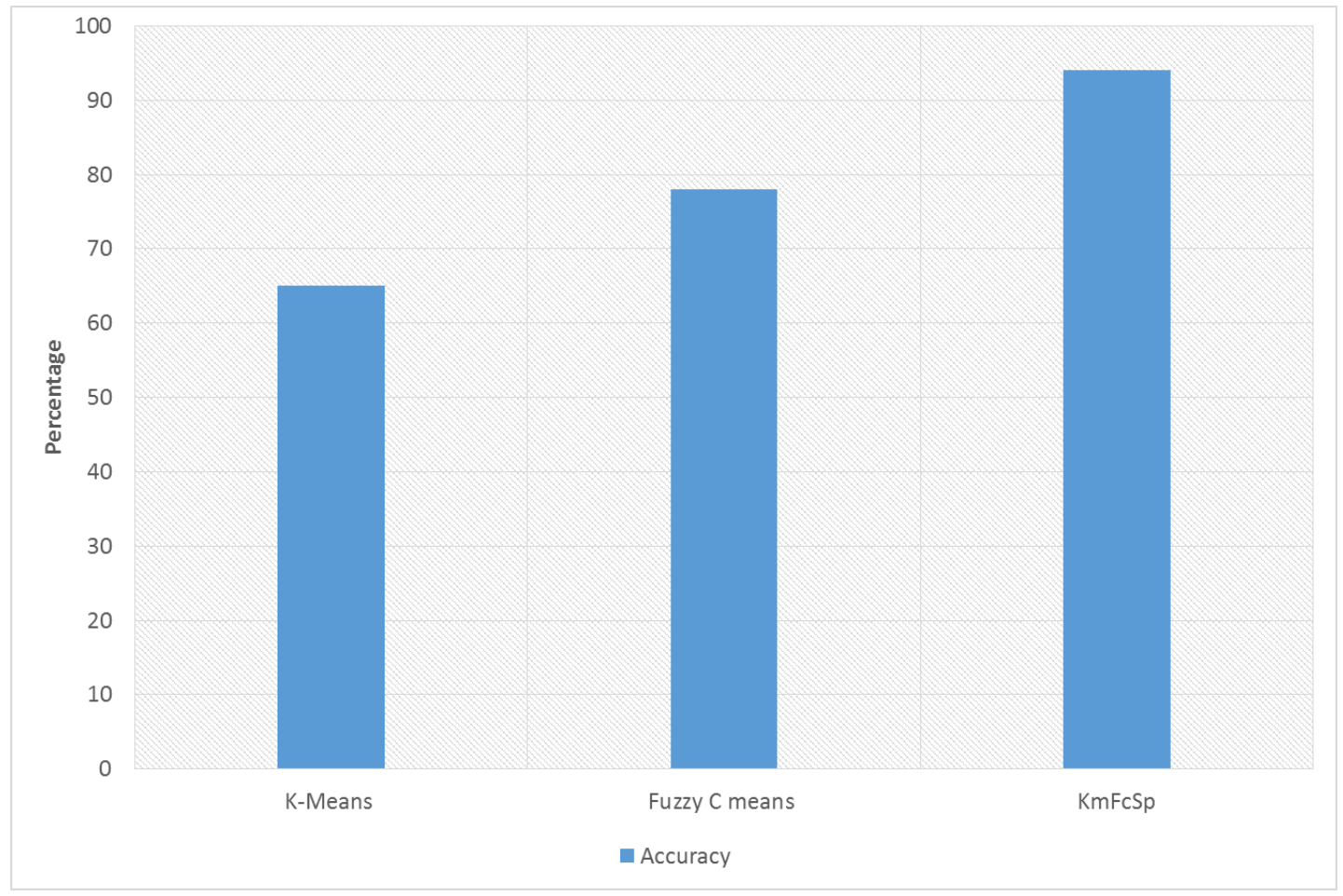

Figure.4 Accuracy

\section{Conclusion}

To have an early detection of brain disorder it is essential to identify the white matter hyper intensities in the brain, the magnetic resonance images of the human brain is acquired and the proposed method integrating the hard and the soft clustering along with the super pixel is devised to segregate the regions of white matter from the rest of the areas. The combined process enabled to have a perfect clustering, proper preservation of actual images information's and obtain optimal results. The evaluation of the results qualitatively and quantitatively shows that the proposed method outperforms the prevailing, the results obtained on the accuracy further proves that the proposed method is highly effective than the prevailing methods of segmenting. 
Journal of Innovative Image Processing (JIIP) (2020)

Vol.02/ No. 01

Pages: $14-24$

https://www.irojournals.com/iroiip/

DOI: https://doi.org/10.36548/jiip.2020.1.002

\section{References}

[1] Heena, A., N. Biradar, and N. M. Maroof. "A novel approach to review various image segmentation techniques." Int. J. Innov. Res. Comput. Commun. Eng. ISO Certif. Organ 32975, no. 2 (2007): 266-269.

[2] Papadopoulos, Symeon, Christos Zigkolis, Giorgos Tolias, Yannis Kalantidis, Phivos Mylonas, Yiannis Kompatsiaris, and Athena Vakali. "Image clustering through community detection on hybrid image similarity graphs." In 2010 IEEE International Conference on Image Processing, pp. 2353-2356. IEEE, 2010.

[3] Brickman, Adam M., Karen L. Siedlecki, Jordan Muraskin, Jennifer J. Manly, José A. Luchsinger, Lok-Kin Yeung, Truman R. Brown, Charles DeCarli, and Yaakov Stern. "White matter hyperintensities and cognition: testing the reserve hypothesis." Neurobiology of aging 32, no. 9 (2011): 1588-1598.

[4] Suganya, R., and R. Shanthi. "Fuzzy c-means algorithm-a review." International Journal of Scientific and Research Publications 2, no. 11 (2012): 1.

[5] Ajala Funmilola, A., O. A. Oke, T. O. Adedeji, O. M. Alade, and E. A. Adewusi. "Fuzzy kcmeans clustering algorithm for medical image segmentation." Journal of Information Engineering and Applications, ISSN 22245782 (2012): 2225-0506.

[6] Wardlaw, Joanna M., Maria C. Valdés Hernández, and Susana Muñoz-Maniega. "What are white matter hyperintensities made of? Relevance to vascular cognitive impairment." Journal of the American Heart Association 4, no. 6 (2015): e001140.

[7] Jeyavathana, R. Beaulah, R. Balasubramanian, and A. Anbarasa Pandian. "A survey: analysis on preprocessing and segmentation techniques for medical images." International Journal of Research and Scientific Innovation (IJRSI) (2016).

[8] Isa, I. S., S. N. Sulaiman, N. Md Tahir, M. Mustapha, and N. K. A. Karim. "A new technique for K-means cluster centers initialization of WMH segmentation." In Proceedings of the International Conference on Imaging, Signal Processing and Communication, pp. 45-49. 2017.

[9] Koresh, M. H., and J. Deva. "Computer vision based traffic sign sensing for smart transport." J. Innovative Image Process.(JIIP) 1, no. 01 (2019): 11-19.

[10] Chandy, Abraham. "A Review On Iot Based Medical Imaging Technology For Healthcare Applications." Journal of Innovative Image Processing (JIIP) 1, no. 01 (2019): 51-60.

[11] Bindhu, V. "Biomedical Image Analysis Using Semantic Segmentation." Journal of Innovative Image Processing (JIIP) 1, no. 02 (2019): 91-101.

[12] Kumar, T. Senthil. "A Novel Method for HDR Video Encoding, Compression and Quality Evaluation." Journal of Innovative Image Processing (JIIP) 1, no. 02 (2019): 71-80. 